\title{
Anti-IL-12/IL-23 treatment for a psoriatic patient with heart failure
}

\author{
Auriemma Matteo, Caponio Chiara, Ruggiero Carlo, \\ Giuliani Federica, Amerio Paolo
}

\begin{abstract}
Introduction: Psoriasis is an inflammatory disorder characterized by a deregulation in the Th1/Th17/Treg cells count and function. A plethora of new drugs called "biologicals" have been developed. Beside TNF-a blockers, new drugs interfering with the IL-23 axis have been introduced. Case Report: We present case of: a 59-year-old high-need psoriatic patient hospitalized for acute heart failure. Physicians decided to withdraw anti-TNF-a therapy because of NYHA class III and the need of cardio-surgery. Surgery was delayed for his general conditions. The patient was referred to our department to establish a new therapy. A treatment with anti-IL-12/IL-23p4o was introduced. Ustekinumab was chosen for the high efficacy rates, the high safety profile and for the reported safety in NYHA class III/IV patients. The rapid general condition improvement enabled the patient to undergo
\end{abstract}

Auriemma Matteo ${ }^{1}$, Caponio Chiara², Ruggiero Carlo ${ }^{3}$, Giuliani Federica ${ }^{3}$, Amerio Paolo ${ }^{4}$

Affiliations: ${ }^{1}(\mathrm{MD}, \quad \mathrm{PhD}$ student), Department of Experimental and Clinical Sciences, Dermatologic Clinic, G. d'Annunzio University, Chieti, Italy; ${ }^{2}$ (MD, resident), Department of Medicine and Aging Science (DMSI), Dermatologic Clinic, G. d'Annunzio University, Chieti, Italy; ${ }^{3}(\mathrm{MD}$, fellow), Department of Medicine and Aging Science (DMSI), Dermatologic Clinic, G. d'Annunzio University, Chieti, Italy; ${ }^{4}(\mathrm{PhD}, \mathrm{MD}$, director), Department of Medicine and Aging Science (DMSI), Dermatologic Clinic, G. d'Annunzio University, Chieti, Italy.

Corresponding Author: Matteo Auriemma, Dermatologic Clinic, SS Annunziata Hospital, Via Dei Vestini, Chieti Scalo (Chieti), 66100, Italy; Phone: +39 (0) 871 358032; Fax: $\quad+39 \quad$ (0) $871 \quad 551057$; E-mail: matteo.auriemma@gmail.com

Received: 04 September 2012

Accepted: 08 December 2012

Published: 01 May 2013 surgery. Conclusion: Anti-TNF-a drugs are contraindicated in NYHA class III/IV patients. In those cases drugs interfering with different cytokine patterns could be of interest. Although anti-IL-12/IL-23p40 has been previously addressed as unsafe in NYHA class III/IV, the clinical practice suggests that this drug can be considered quite safe. Currently the role of antiIL-12/IL-23p40 on cardiovascular diseases is still an open question and deserves further investigations. Treatment with anti-IL-12/IL$23 p 40$ can be considered a good option in NYHA class III/IV patients and in high- need psoriasis patients.

Keywords: Psoriasis, MACE, Anti-IL-12/IL23p4o, Ustekinumab

$$
* * * * * * * * *
$$

Matteo A, Chiara C, Carlo R, Federica G, Paolo A. AntiIL-12/IL-23 treatment for a psoriatic patient with heart failure. International Journal of Case Reports and Images 2013;4(5):266-269.

$$
* * * * * * * *
$$

doi:10.5348/ijcri-2013-05-309-CR-6

\section{INTRODUCTION}

Psoriasis is a systemic inflammatory disorder associated with several comorbidities (metabolic, cardiac and psychiatric). Several immunological mechanisms are involved in pathogenesis of psoriasis. A deregulation of Th1, Th17 and regulatory T cells (Tregs) results in persistent T-cell activation and high expression level of proinflammatory cytokines such as tumor necrosis factor- $\alpha$ (TNF- $\alpha$ ), interferon- $\gamma$ (IFN- $\gamma$ ) and interleukins like IL-12, IL-17 and IL-23 and their receptors [1]. 
A plethora of new drugs called "biologicals" have been developed. These interfere with the pathological mechanism of psoriasis, directly blocking or antagonizing several cytokines. Two main class exist: TNF- $\alpha$-blockers and anti-IL-12/IL-23.

Ustekinumab is the latest "biological" approved by FDA for the treatment of moderate to severe plaque psoriasis. The main target of this drug is the IL-12/IL23p40 subunit thus interfering with Th17 maintenance and inflammation progression [2]. High efficacy rates coupled with a good safety profile are the main advantages of ustekinumab. Anti-TNF- $\alpha$ agents have been addressed as unsafe in NYHA class III/IV patients and are contraindicated in these conditions. However, some studies in rheumatoid arthritis (RA) patients in NYHA class III/IV treated with biologics, seem to suggest a possible role of those drugs not only in controlling RA or psoriasis but also in ameliorating the cardiologic condition. New "biologic" drugs (e.g. antiIL-12/IL-23p40) does not seem to have a negative impact on NYHA class III/IV patients and are currently described as a good option in the treatment of high need patients with cardiac failure or cardiac failure predisposing conditions. Clinical surveillance is needed to assess the safety of the drugs in high need patients. We describe this paradigmatic case to underline the importance of ustekinumab in patients with cardiac diseases.

\section{CASE PRESENTATION}

In December 2011, a 59-year-old male was referred to the outpatient clinic with a severe psoriatic erythroderma. The patient suffered from psoriasis since the age of 18 years. He had no familiar history of psoriasis. His past medical history revealed deep vein thrombosis (two episodes treated with thrombectomy), epilepsy, type II diabetes, chronic renal failure (since year 2000) and hepatosteatosis. He quit smoking 25 years ago and did not drink alcohol. No drug allergies were reported.

Since first presentation of psoriasis, the patient underwent topical treatments, several UVB-narrow band (NB) courses with good responses. When his psoriasis worsened he was treated with two retinoid courses, cyclosporine $(4 \mathrm{mg} / \mathrm{kg} /$ day $)$ and methotrexate (15 mg/week) for several courses with only temporary improvement and worsening of the hepato-steatosis and serum lipid levels.

At this time (about six years ago) his psoriasis area and severity index score was 21 and he begun the first monoclonal antibody therapy. After nine months of therapy infliximab was withdrawn because of lack of efficacy. For a new psoriasis flare up, the patient was switched to a second monoclonal antibody, etanercept, $50 \mathrm{mg}$ once-a-week achieving a significant amelioration of skin presentation (PASI score 90 after three months).

At this time his daily therapy included bisoprolol 125 $\mathrm{mg}$, pantoprazole $20 \mathrm{mg}$, potassium canrenoate $100 \mathrm{mg}$, allopurinol $300 \mathrm{mg}$, fenobarbital $100 \mathrm{mg}$, furosemide
$125 \mathrm{mg}$ and acetylsalicylic acid $100 \mathrm{mg}$ daily and metformin $500 \mathrm{mg}$ twice daily.

In August 2010, while still under etanercept treatment, he was admitted to a secondary hospital for an acute heart failure secondary to bronchopneumonia. During hospitalization a diagnosis of severe heart insufficiency due to valvular heart disease (aortic and mitral valve) was made. Because of the cardiopulmonary (NYHA class III) conditions, and the necessity of surgery (aorto-mitral valve replacement), physicians decided to withdrawn anti-TNF therapy as suggested in different international guidelines. However, due to the patient's general inflammatory condition, surgery was postponed.

The patient was then referred to our department. At this time PASI score was 31.3 and BSA was $76 \%$ (Figure 1). Blood tests were representative for a severe inflammatory state: ESR $96 \mathrm{~mm} / 1 \mathrm{st} \mathrm{hr}$ CRP 11.70 mg/dL, ANA 1/160 (homogeneous pattern), ASLO 212 $\mathrm{IU} / \mathrm{mL}$, RBC count $3.19 \times 10^{6} / \mathrm{mm}^{3} ; \mathrm{Hb} 9.5 \mathrm{~g} / \mathrm{dL}$, het $30.5 \%$; TLC $13.0 \times 10^{3} / \mathrm{mm}^{3}$ and lymphocyte count $1.15 \times 10^{3} / \mathrm{mm}^{3}$. Chest X-ray was unremarkable: Mantoux test (PPD) and quantiferon were all negative.

After a through evaluation of the immunological, serological and cardiac condition it was decided to start a new treatment with ustekinumab subcutaneously at standard dose (45 mg every 4 weeks for the first month and then every three months) (patient's height $172 \mathrm{~cm}$; weighs $68 \mathrm{~kg}$; BMI $22.99 \mathrm{~kg} / \mathrm{m}^{2}$ ). In January 2012, after 4 weeks, inflammatory markers lowered significantly (CRP $6.79 \mathrm{mg} / \mathrm{dL}$, ESR $76 \mathrm{~mm}$ ). PASI score decreased to 19.5/1st $\mathrm{hr}$ and BSA was 30\% (Figure 2), leading to a marked improvement in general condition. A second subcutaneous injection of ustekinumab was then administered. The patient has reported no infections or any other complications and his cardiac condition were stable although still severe. Due to the high need of this

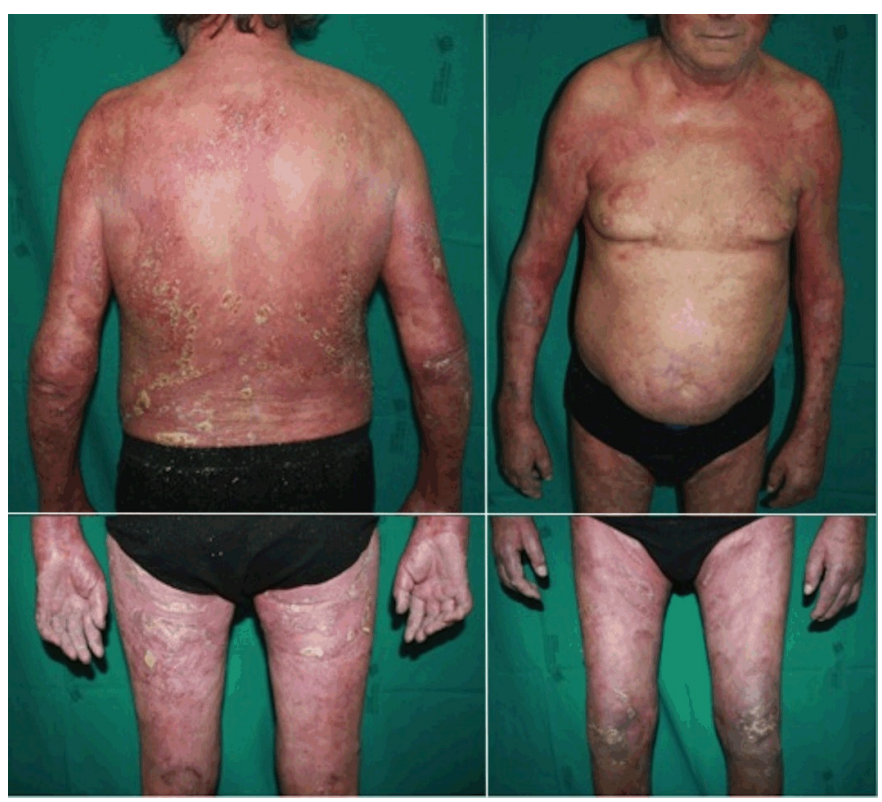

Figure 1: Sub-erythrodermic psoriasis. Psoriasis area and severity index was 31.3 and BSA was $76 \%$. After discontinuation of the first monoclonal antibody. 


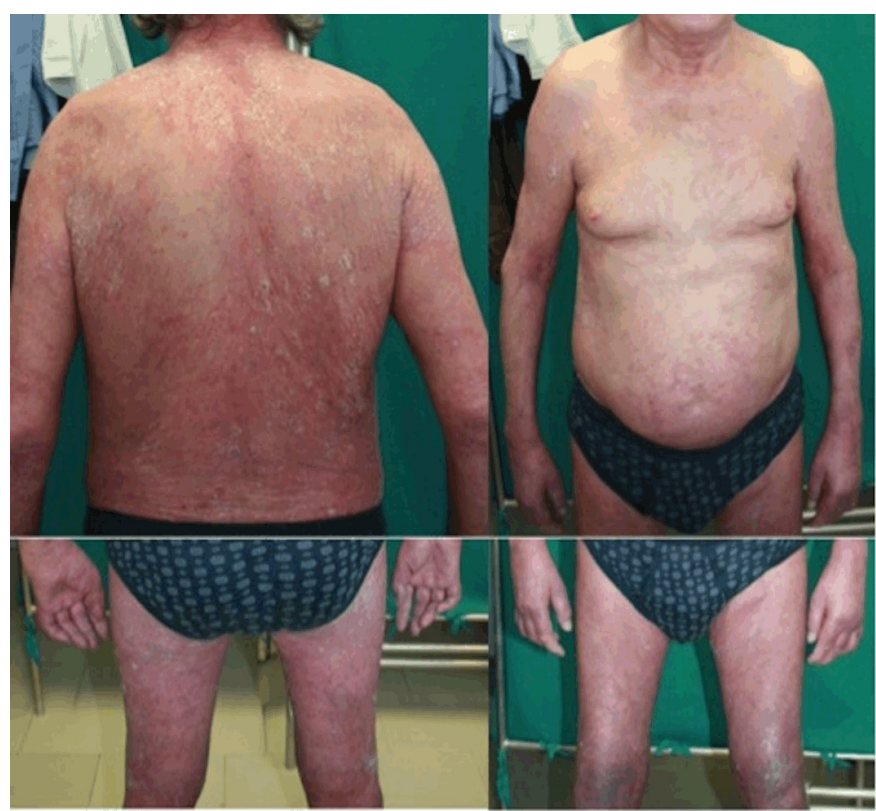

Figure 2: Clearance of psoriatic lesions. psoriasis area and severity index score 19.5; BSA 30\% four weeks after IL-12/IL23 p40 administration.

patient an additional check-up was done on February 2012. A more pronounced improvement of PASI and BSA score was achieved ( 8.3 and $15 \%$ respectively) together with further lowering of inflammatory markers (ESR $65 \mathrm{~mm} / 1 \mathrm{st} \mathrm{hr}, \mathrm{CRP} 4.81 \mathrm{mg} / \mathrm{dL}$ ). The patient underwent surgery for heart-valve replacement in February 2012 and at the last follow-up in April 2012 his general conditions were good and psoriasis was under control (PASI score 8).

\section{DISCUSSION}

Many reports show that IL-12 and IL-23 play a key roles in the development of psoriasis, inducing INF and TNF producing $\mathrm{T}$ cells and stabilizing Th17 phenotype, determining the secretion of cytokines responsible for the prototypical skin modifications in the psoriatic plaques [3, 4].

Ustekinumab, blocking p40 subunit of IL-12/IL-23, interferes with this inflammatory pathways.

Ustekinumab has been approved in Europe for the treatment of moderate to severe psoriatic patients. Two randomized, double-blind, placebo controlled Phase III trials have demonstrated ustekinumabs excellent PASI achievements and good safety profile. Serious adverse events included severe infections, malignancies and cardiovascular diseases [5].

Treatment of severe psoriasis in patients with cardiovascular morbidity and with cardiac insufficiency has been always a challenge, especially because treatment with anti TNF is contraindicated in patients with NYHA class III-IV cardiac insufficiency.

However, some reports investigated the safety of biological drugs for inflammatory condition in NYHAclass-III-IV-patients. Additionally, in the past decade a link between cardiovascular events and inflammatory autoimmune disorders like psoriasis, RA and crohn's disease has been hypothesized and investigated. Unfortunately preliminary reports on anti-IL-12/IL23p40 (ustekinumab and briakinumab) safety in the treatment of psoriasis patients showed a numerical excess of major adverse cardiovascular events (MACE) [6].

More recently a meta-analysis of 22 randomized controlled trials evaluating cardiovascular safety of antiTNF (etanercept, adalinumab and infliximab) and antiIL-12/IL-23p40 (ustekinumab) in the treatment of psoriasis showed that both "classes of biological drugs" did not significantly increase the risk of MACEs [7]. A little number of cardiovascular events possibly associated with the general increased cardiovascular risk were observed in some patients. Another recent paper also reported that cardiac-adverse-events-rates in patients treated for four years with ustekinumab were similar to those of the general population, indicating a good safety profile of the drug [5]. This body of literature seems to address the importance of inflammation in the development of cardiovascular disease (CVD) and suggests that therapies aimed at reducing disease activity in chronic inflammatory diseases may also have a positive impact on CVD risk by reducing the burden of systemic inflammation and progression of atherosclerosis. In psoriasis patients the reduction of inflammation is usually associated with serum C-reactive protein (a known risk factor for CVD) reductions, particularly in the case of using anti-IL12/IL-23 agents [8].

However, the role of IL-12 or IL-23 in the pathogenesis of cardiac failure is still under investigation. In case their role would be confirmed, more accurate studies on anti TNF- $\alpha$ and anti IL-12/IL23p40 MACE safety profile would be mandatory to better understand the safety profile of those biological drugs in psoriasis patients with cardiac comorbidities.

\section{CONCLUSION}

The role of anti TNF- $\alpha$ and anti IL-12/IL-23p40 on cardiovascular diseases is still an open question. Although their is lack of exhaustive data on there is safety of biological drug use in case of NYHA class III-IV psoriasis patients, ustekinumab can be used due to the high therapeutic index, rapid mode of action and the good safety profile. However, anti-IL-12/IL-23p40 and anti-TNF- $\alpha$ should be better assessed for the use in NYHA class III-IV-psoriasis patients in which more traditional therapies failed to gain good results in reducing PASI and Dermatology Life Quality Index (DLQI) score.

$$
* * * * * * * * *
$$

\section{Author Contributions}

Auriemma Matteo - Substantial contributions to conception and design, Acquisition of data, Analysis and interpretation of data, Drafting the article, Revising it 
critically for important intellectual content, Final approval of the version to be published

Ruggiero Carlo - Acquisition of data, Drafting the article, Revising it critically for important intellectual content, Final approval of the version to be published Giuliani Federica - Acquisition of data, Drafting the article, Revising it critically for important intellectual content, Final approval of the version to be published Amerio Paolo - Acquisition of data, Drafting the article, Revising it critically for important intellectual content, Final approval of the version to be published

\section{Guarantor}

The corresponding author is the guarantor of submission.

\section{Conflict of Interest}

Authors declare no conflict of interest.

\section{Copyright}

(C) Auriemma Matteo et al. 2013; This article is distributed under the terms of Creative Commons Attribution 3.0 License which permits unrestricted use, distribution and reproduction in any means provided the original authors and original publisher are properly credited. (Please see www.ijcasereportsandimages.com /copyright-policy.php for more information.)

\section{REFERENCES}

1. Steinman L. A rush to judgment on Th17. J Exp Med 2008;205(7):1517-22.

2. Cingoz O. Ustekinumab. MAbs 2009;1(3):216-21.

3. Nickoloff BJ, Xin H, Nestle FO, Qin JZ. The cytokine and chemokine network in psoriasis. Clin Dermatol 2007;25(6):568-73.

4. Toichi E, Torres G, McCormick TS, et al. An anti-IL12p40 antibody down-regulates type 1 cytokines, chemokines, and IL-12/IL-23 in psoriasis. J Immunol 2006;177(7):4917-26.

5. Reich K, Papp KA, Griffiths CE, et al. An update on the long-term safety experience of ustekinumab: results from the psoriasis clinical development program with up to four years of follow-up. J Drugs Dermatol 2012;11(3):300-12.

6. Ryan C, Leonardi CL, Krueger JG, et al. Association between biologic therapies for chronic plaque psoriasis and cardiovascular events: a meta-analysis of randomized controlled trials. JAMA 2011;306(8):864-71.

7. Reich K, Papp KA, Griffiths CE, et al. An update on the long-term safety experience of ustekinumab: results from the psoriasis clinical development program with up to four years of follow-up. J Drugs Dermatol 2012;11(3):300-12.

8. Toedter GP, Blank M, Lang Y, Chen D, Sandborn WJ, de Villiers WJ. Relationship of C-reactive protein with clinical response after therapy with ustekinumab in Crohn's disease. Am J Gastroenterol 2009;104(11):2768-73.
Access full text article on other devices

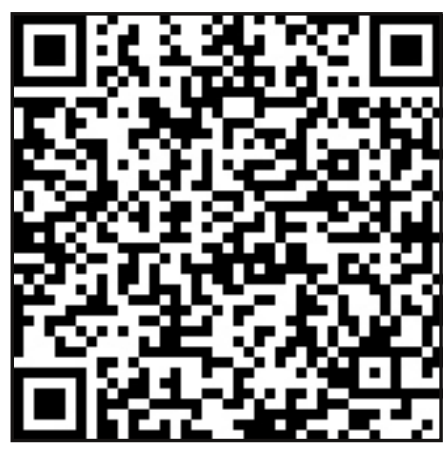

Access PDF of article on other devices

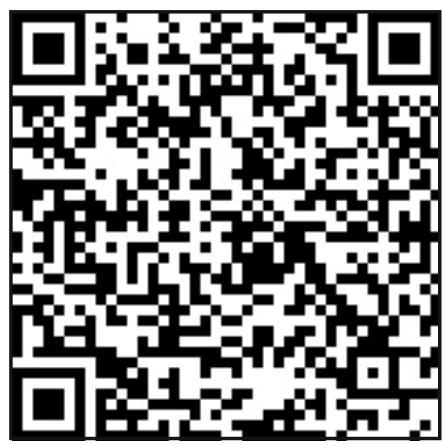

